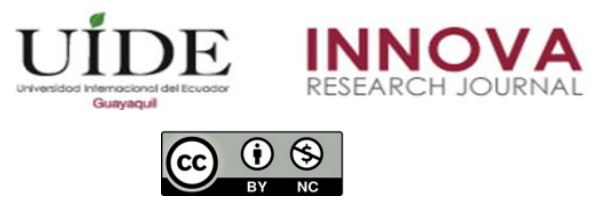

INNOVA Research Journal, ISSN 2477-9024

(Septiembre-Diciembre 2020). Vol. 5, No.3.2 pp. 295-308

DOI: https://doi.org/10.33890/innova.v5.n3.2.2020.1612

URL: http://revistas.uide.edu.ec/index.php/innova/index

Correo: innova@uide.edu.ec

\title{
Análisis de la Viabilidad para la Implementación de Vehículo Eléctrico que Preste Servicio de Taxi en la Ciudad de Cuenca
}

\section{Analysis of the Feasibility for the Implementation of an Electric Vehicle that Provides Taxi Service in Cuenca City}

\author{
Paul Wilfrido Méndez Torres \\ Universidad Politécnica Salesiana, Ecuador \\ Manuel Fernando Gómez Berrezueta \\ https://orcid.org/0000-0002-2914-2892 \\ Alex Fernando Llerena Mena \\ https://orcid.org/0000-0002-3847-7141 \\ Universidad International del Ecuador, Ecuador \\ Autor para correspondencia: pmendez@ups.edu.ec; magomezbe@uide.edu.ec; \\ alllerename@uide.edu.ec
}

Fecha de recepción: 09 de octubre de 2020 - Fecha de aceptación: 23 de diciembre de 2020

\begin{abstract}
Resumen
El transporte es un elemento esencial para el crecimiento socioeconómico, sin embargo, su desarrollo genera una serie de efectos tales como la congestión, el ruido, la emisión de sustancias nocivas al medio ambiente, el agotamiento de los recursos no renovables. Uno de los factores que inciden en la reducción de la contaminación al medioambiente es la sustitución de los vehículos de combustible convencional por otros tipos de sistemas de propulsión, incluidos los motores eléctricos. La introducción de vehículos eléctricos puede convertirse en el elemento clave en el desarrollo de la estrategia de transporte sostenible en las zonas urbanas, la demanda de estos vehículos debe ser impulsada por los propietarios de la flota de vehículos, especialmente por las empresas. Los propietarios de taxis convencionales en la ciudad de Cuenca que deben renovar sus unidades, según la AFUT- Asociación Frente Unido de Taxistas-, son escépticos sobre la renovación con unidades eléctricas que proponen las autoridades locales, debido principalmente a la incertidumbre sobre la fiabilidad y rentabilidad que ofrece este tipo de vehículo, las cuales deben ser por lo menos igual a las de un vehículo con motor de combustión interna. En esta investigación se aplicó una metodología de investigación experimental mediante el uso de un software de computadora EMOLAB y un vehículo eléctrico Kia Soul EV para obtener una base de datos con información que describe el comportamiento del vehículo eléctrico. Aplicando diseños de experimentos factorial (DOE) se utilizó las variables que tienen más influencia sobre la autonomía y consumo energético del vehículo eléctrico para su evaluación.
\end{abstract}

Palabras clave: Vehículos eléctricos; transporte; ciclos de conducción; autonomía. 


\begin{abstract}
Transportation is an essential element for socioeconomic growth; however, its development generates a series of effects such as congestion, noise, the emission of harmful substances into the environment, the depletion of non-renewable resources. One of the factors that affect the reduction of pollution to the environment is the replacement of conventional fuel vehicles by other types of propulsion systems, including electric motors. The introduction of electric vehicles can become the key element in the development of the sustainable transport strategy in urban areas, the demand for these vehicles must be driven by the owners of the vehicle fleet, especially by companies. The owners of conventional taxis in the city of Cuenca who must renew their units, according to the AFUT- Asociación Frente Unido de Taxistas-, are skeptical about the renewal with electric units proposed by local authorities, mainly due to the uncertainty about the reliability and profitability offered by this type of vehicle, which must be at least equal to those of a vehicle with an internal combustion engine. In this research, an experimental research methodology was applied by using EMOLAB computer software and a Kia Soul EV electric vehicle to obtain a database with information that describes the behavior of the electric vehicle. Applying factorial experiment designs (DOE), the variables that have the most influence on the autonomy and energy consumption of the electric vehicle were used for their evaluation.
\end{abstract}

Keywords: Electric vehicles; transport; driving cycles; autonomy.

\title{
Introducción
}

Un vehículo eléctrico es aquel que usa energía almacenada en forma química en una o varias baterías que alimentan un motor, el cual transforma la energía eléctrica en mecánica, para dar tracción a las ruedas del vehículo y este se pueda desplazar. La energía cinética de las ruedas en movimiento también puede ser usada, a través de un sistema regenerativo, para cargar la batería. De otra forma y siempre que la infraestructura eléctrica lo permita, se puede conectar el vehículo a la red eléctrica para recargar en forma total la batería (R. G. Déniz, 2013) (J. L. D. Fernández, 2013) (J. D. T. Sarmiento, 2015).

Cuando se escucha acerca del vehículo eléctrico la sociedad en general tiende a pensar que se trata de un invento tecnológico reciente, no obstante, su investigación y desarrollo, aunque lento, datan de hace más de un siglo; de hecho, este se desarrolló más pronto que el automóvil de combustión interna que predomina actualmente. Y aunque fue por un corto periodo de tiempo, a inicios del XX, el vehículo eléctrico circulaba por los caminos de algunas ciudades junto a otros vehículos de tracción animal, de vapor y combustión, llegando incluso a competir contra este último por la supremacía en la propulsión de automóviles, hasta que se impuso el vehículo con motor de combustión interna.

Sin embargo, no se puede desconocer los problemas medioambientales que atraviesa el planeta, mismos que se deben principalmente a la acumulación en el aire de varios productos tóxicos que dañan la salud de humanos, animales, plantas y alteran los ecosistemas de forma negativa. Entre los diversos agentes que contribuyen a la contaminación atmosférica se tiene el motor de combustión de un automóvil, que desprende a la atmósfera productos nocivos como: óxidos nitrosos $\left(\mathrm{NO}_{\mathrm{X}}\right)$, monóxido de carbono $(\mathrm{CO})$, dióxido de carbono $\left(\mathrm{CO}_{2}\right)$, hidrocarburos no quemados (HC) y partículas sólidas además de otros residuos en menor medida. Para reducir la 
contaminación atmosférica, aprovechar las ventajas que supone la transformación de la matriz energética que está viviendo el país y reducir la dependencia de fuentes de energía contaminantes como el petróleo, es necesario hacer uso de medios de transporte como el vehículo eléctrico.

\section{Revisión Literaria}

Barcelona (España)

La ciudad de Barcelona es una de las pioneras en España en cuanto a movilidad sostenible e inició estrategias para impulsar vehículos eléctricos desde el año 2009 con lo cual se consiguió implementar los primeros taxis eléctricos en 2014 y hasta 2015 se construyeron un total de 336 puntos de recarga entre todos los promotores (González, 2015) y cabe destacar que 27 son puntos de recarga rápida de acceso público, de estos, 17 se encuentran en la ciudad y 10 en los municipios metropolitanos (AMB, 2017). Actualmente en Barcelona circulan aproximadamente 10500 taxis, de ellos solo 20 son eléctricos, entre los que se tiene: trece BYD e6, un Nissan LEAF y seis Nissan e-NV200 (AEDIVE, 2017); dichos modelos no han sido tan exitosos a pesar de las ayudas estatales y de la Generalitat de Catalunya que han recibido los propietarios según (González, 2015).

\section{Bogotá (Colombia)}

En Bogotá la iniciativa de implementar taxis eléctricos empezó mediante el Decreto 677 del año 2011 en el que la Administración Distrital expresaba la necesidad de elaborar una política de movilidad eléctrica que sea eficiente y que no contamine Bogotá, donde circulan más de 50000 taxis. En un principio se esperaba que el "plan piloto de movilidad eléctrica en el transporte público", apoyado a demás por otras entidades y empresas privadas, arranque con 50 taxis eléctricos (Ambiente, 2017). Los primeros empezaron a circular desde el año 2013, el vehículo elegido fue el BYD e6 y lo cierto es que a julio del 2017 se encuentran circulando 40 unidades con alta probabilidad de que el proyecto finalmente fracase debido a la falta de respaldo de la administración actual y de las empresas privadas que se están desvinculando de la iniciativa, la falta de puntos de recarga, los cuales solo se han implementado 5 en toda la ciudad y ninguno es de recarga rápida a más de un deficiente servicio posventa de la marca BYD, la cual está representada en Colombia por la empresa automotriz Praco Didacol (Cárdenas, 2017) (COMUNICADOS.CO, 2017).

\section{Ámsterdam (Países Bajos)}

Es quizás el ejemplo más representativo en cuanto a movilidad eléctrica de todo el mundo ya que en esta ciudad se puede encontrar un punto de recarga para vehículos eléctricos cada 200 metros y para 2015 ya contaba con 1250 puntos de recarga públicos y el objetivo es alcanzar los 4000 puntos de recarga para 2018. La implementación de taxis eléctricos empezó en el 2011 y para abril del 2015 en Ámsterdam circulaban 170 taxis eléctricos de la marca Nissan, modelos LEAF y e-NV200, operados por tres compañías: Taxi Electric, Connexxion y TCA. Por otro lado, en 2014 se implementaron cerca de 200 taxis eléctricos Tesla Model S para el aeropuerto de Schiphol. El apoyo que reciben los propietarios por parte del estado es importante en cuanto a financiamiento y subvenciones, así mismo se han desarrollado lo que se conoce como paradas 
verdes, donde los taxis eléctricos tienen prioridad cuando hay colas por carreras (movilidadeléctrica.com, 2017) (Mourik, 2017).

Vehículo eléctrico en Ecuador

En referencia a lo indicado por (MCPEC, 2017), entre los objetivos estratégicos planteados por el Gobierno se encuentra producir en Ecuador vehículos eléctricos y sus baterías, esto con la finalidad de aprovechar el cambio de la matriz energética que está viviendo el país y reducir la dependencia del petróleo. Es así que el MCPEC -Ministerio Coordinador de Producción, Empleo y Competitividad- firmó un convenio en marzo de 2015 con Nissan, Renault, Kia y BYD denominado "Convenio Marco para la Promoción, Comercialización, y Perspectivas de Fabricación de Baterías y Vehículos Eléctricos en la República del Ecuador”.

El BEV no ha tenido una participación significativa en el mercado nacional, la tabla 1 y la tabla 2 son elaboradas con datos obtenidos de (MUNICPAL, 2016) así lo reflejan.

\section{Tabla 1}

Venta de vehículos eléctricos en principales provincias del año 2016

\begin{tabular}{ccc}
\hline Provincia & Unidades & Participación (\%) \\
\hline Pichincha & 62 & 56.88 \\
Guayas & 20 & 18.35 \\
Galápagos & 9 & 8.26 \\
Loja & 9 & 8.26 \\
Tungurahua & 4 & 3.67 \\
Otras & 5 & 4.59 \\
\hline Total & $\mathbf{1 0 9}$ & $\mathbf{1 0 0}$ \\
\hline
\end{tabular}

\section{Tabla 2}

Venta de vehículos eléctricos por marca y modelos del año 2016

\begin{tabular}{ccc}
\hline Marca & Modelo & Unidades \\
\hline Kia & SOUL AC 5P 4X2 TA EV & 53 \\
Renault & TWIZY URB X09 MOKA AC 2P 4X2 TA EV & 23 \\
Dayang & DY-GD04A AC 2P 4X2 TA EV & 15 \\
Dayang & DY-GD02B AC CS 4X2 TA EV & 7 \\
Renault & TWIZY TEC X09TEC80 2P 4X2 TA EV & 6 \\
Volkswagen & E-GOLF BE11B1 AC 5P 4X2 TA EV & 1 \\
Dayang & DY-GD04A AC 4P 4X2 TA EV & 2 \\
Renault & TWIZY CARGO X09CAR80 2P 4X2 TA EV & 1 \\
Renault & TWIZY URB X09 MOKA 2P 4X2 TA & 1 \\
\hline
\end{tabular}


Analizando la tabla 2 se puede apreciar que el Kia Soul EV es el vehículo eléctrico más vendido del Ecuador debido a su precio, más accesible con respecto a otros EVs, se lo puede adquirir desde 30990 USD y tiene una autonomía según la marca de $200 \mathrm{~km}$ aproximadamente; el Renault Twizy el segundo más vendido desde 14990 USD en la versión pasajero y 15990 USD en la versión carga, es más económico que el Soul EV sin embargo a diferencia de este que es un monovolumen, el Twizy es un biplaza y cuenta con $100 \mathrm{~km}$ de autonomía según Renault. Por su parte el Volkswagen e-GOLF todavía no ha sido comercializado por la marca en Ecuador y el precio en Alemania del modelo 2017 es de aproximadamente 42661 USD. Por otro lado, Dayang, fabricante de motocicletas todavía no es una marca conocida en el mercado nacional.

A pesar de que Nissan fue parte del convenio para la promoción de vehículos eléctricos en Ecuador y que el Nissan Leaf es el vehículo eléctrico más vendido del mundo, en Ecuador tampoco se ha comercializado, seguramente debido a su precio que en Europa está entre 3499837611 USD. Por su parte BYD ha ingresado al mercado nacional este año 2017 con el BYD e5 cuyo precio es de 34500 USD aproximadamente y autonomía de $350 \mathrm{~km}$ según la marca. El BYD e5 ha ingresado con 30 vehículos para completar la primera flota del país integrada únicamente por taxis eléctricos en Loja.

\section{Metodología}

La CNTTTSV (Comisión Nacional de Transporte Terrestre, Tránsito y Seguridad Vial) define el servicio de transporte comercial de pasajeros en taxi como (ANT, Resolución No. 006B-DIR-2009-CNTTTSV, 2009): “...el que se presta a terceras personas a cambio de una contraprestación económica, siempre que no sea servicio de transporte masivo o colectivo, en vehículos de color amarillo denominados TAXI, organizados en operadoras legalmente constituidas...".

En lo referente a la modalidad o clase de servicio, la CNTTTSV dice que se prestará como convencional y ejecutivo.

El servicio convencional consiste en (ANT, Resolución No. 006-B-DIR-2009CNTTTSV, 2009):

“...el traslado de personas desde un lugar a otro dentro del área urbana autorizada para su operación, en vehículos automotores de color amarillo, equipados para el transporte de personas, con capacidad de hasta cinco pasajeros incluido el conductor... Los vehículos que prestan este servicio podrán operar como ambulantes, a pedido expreso de los pasajeros en las calles." 2009):

El servicio ejecutivo consiste en (ANT, Resolución No. 006-B-DIR-2009-CNTTTSV,

“...el traslado exclusivo de personas en forma segura dentro de su ámbito de operación, siempre que no sea servicio de transporte masivo o colectivo, en vehículos de color amarillo, y que se lo contratará única y exclusivamente a través de los medios de telecomunicación establecidos y autorizados por cada uno de ellos." 
Así mismo, en la resolución de la ANT y su reforma (ANT, Resolución No. 053-DIR2015-ANT, 2015), mencionan que el tiempo de vida útil de las unidades es de 15 años en el servicio convencional y 10 años en el servicio ejecutivo y que en ambas modalidades el cobro está controlado por taxímetro; por otro lado, para el análisis de viabilidad se considerará que el Kia Soul EV opera como taxi convencional debido a que este servicio tiene más demanda que el ejecutivo.

En cuanto a las características del sector de taxis en Cuenca, es importante destacar algunos datos estadísticos, el número de pasajeros que ocupa un vehículo: 2 pasajeros representan el 51\%, 4 pasajeros representan el $37 \%$ y más de 4 pasajeros que representa el $12 \%$. En la figura 1 se aprecia que los viajes en taxi ocurren entre las 9 y las 11 de la mañana y que la mayoría de los viajes dura de 15 a 30 minutos.

\section{Figura 1}

Duración del viaje en taxi.

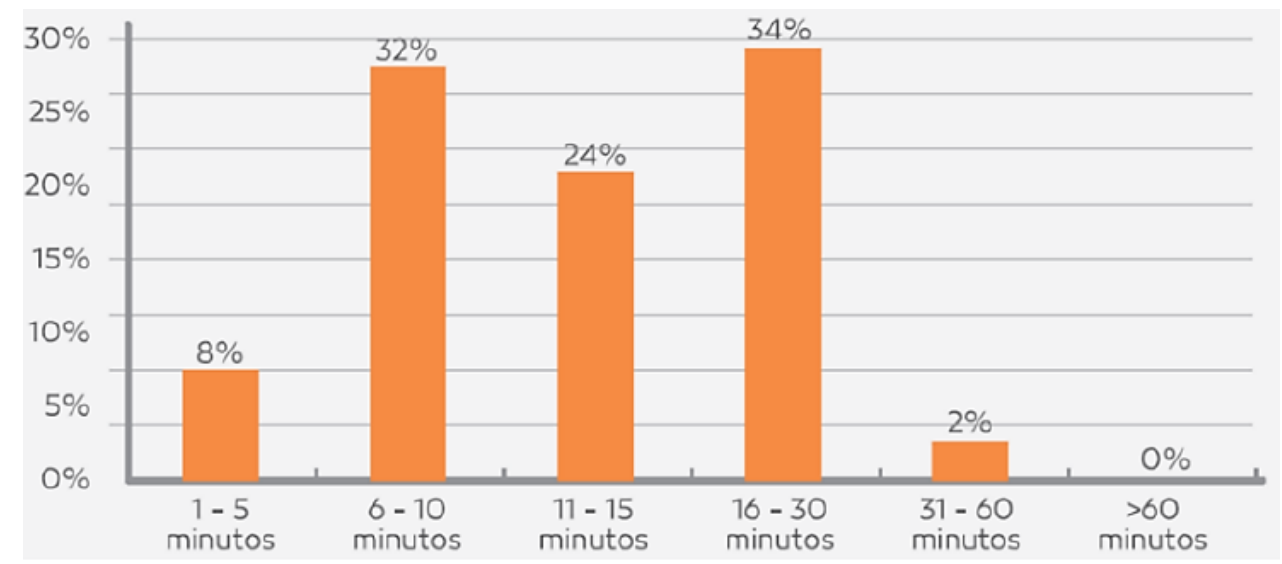

De acuerdo con Moncayo (Moncayo, 2016), en su estudio estadístico se determinó que la mayoría de los taxis en Cuenca recorre entre 134.58 y 272.08 kilómetros diarios, aunque en promedio la distancia recorrida diariamente es de 200.29 kilómetros, este valor representa el indicador Kilómetros Vehículo Recorrido.

\section{Adquisición de datos}

Se describe el comportamiento del vehículo de pruebas a partir de los ciclos típicos de conducción para los vehículos de la Unión de Taxistas del Azuay, con las diferentes fuerzas y resistencias que se oponen al movimiento del vehículo, una vez definidos los parámetros se procederá a la realización de pruebas experimentales para determinar el consumo y la autonomía real del vehículo eléctrico operando bajo la modalidad taxi convencional en la ciudad de Cuenca.

\section{Descripción del protocolo de muestreo}


El proceso de adquisición de datos se realizó bajo diferentes condiciones de muestreo, como el peso y la pendiente, variables que afectan directamente al consumo energético del vehículo y por lo tanto en la autonomía de este.

Vehículo eléctrico de pruebas Kia Soul EV

Este proyecto de análisis de viabilidad para la implementación del taxi eléctrico se desarrollará, con el Kia Soul EV, que es el vehículo eléctrico de mayor demanda en el mercado nacional por su precio más asequible y además se dispone de una unidad (figura 2) en la Universidad Politécnica Salesiana sede Cuenca.

\section{Figura 2}

Kia Soul EV del Proyecto "Movilidad Verde UPS"

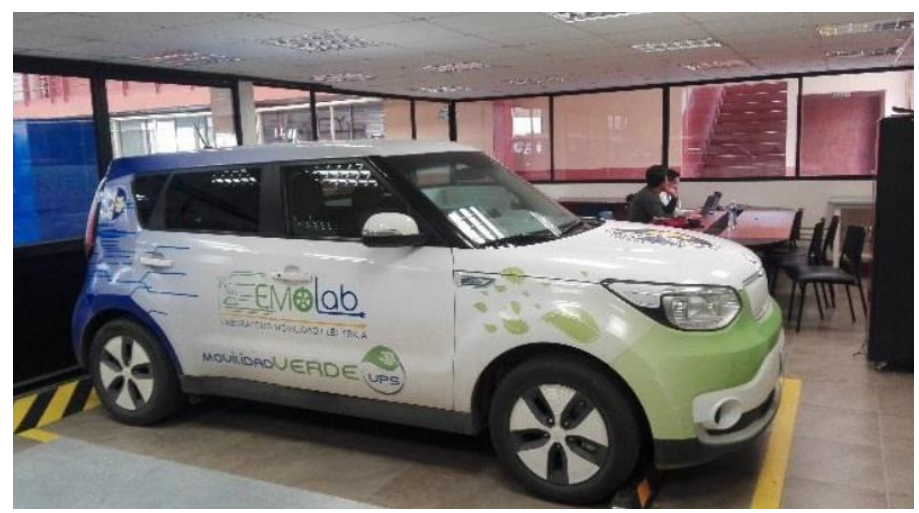

\section{Equipos para la medición de los parámetros de funcionamiento del vehículo}

Mediante metodología de pruebas experimentales se realiza un proceso de adquisición de datos por medio de un programa de ordenador software llamado EMOLAB V2.0.1 (Gonzalez, 2017), con un ciclo típico de conducción para los taxis de la ciudad de Cuenca, en el cual interviene variables que afectan en la autonomía del vehículo eléctrico (pendiente, velocidad mínima, velocidad máxima, distancia de recorrido, aceleraciones, desaceleraciones y modo de conducción).

El programa EMOLAB V2.0.1, cuya interfaz se ve en la figura 3, fue desarrollado en LabVIEW y es un software de ingeniería que sirve como traductor de la información emitida por el escáner automotriz OBD II conectado al vehículo eléctrico y enlazado al software mediante bluetooth. Este software proporciona 72 variables de estudio tales como la temperatura, voltaje y corriente de: la batería, las celdas que la conforman, los módulos y el motor eléctrico; además de otras como el estado de carga, velocidad y aceleración del vehículo. 
Figura 3

Pantalla principal del Software EMOLAB V2.0.1.

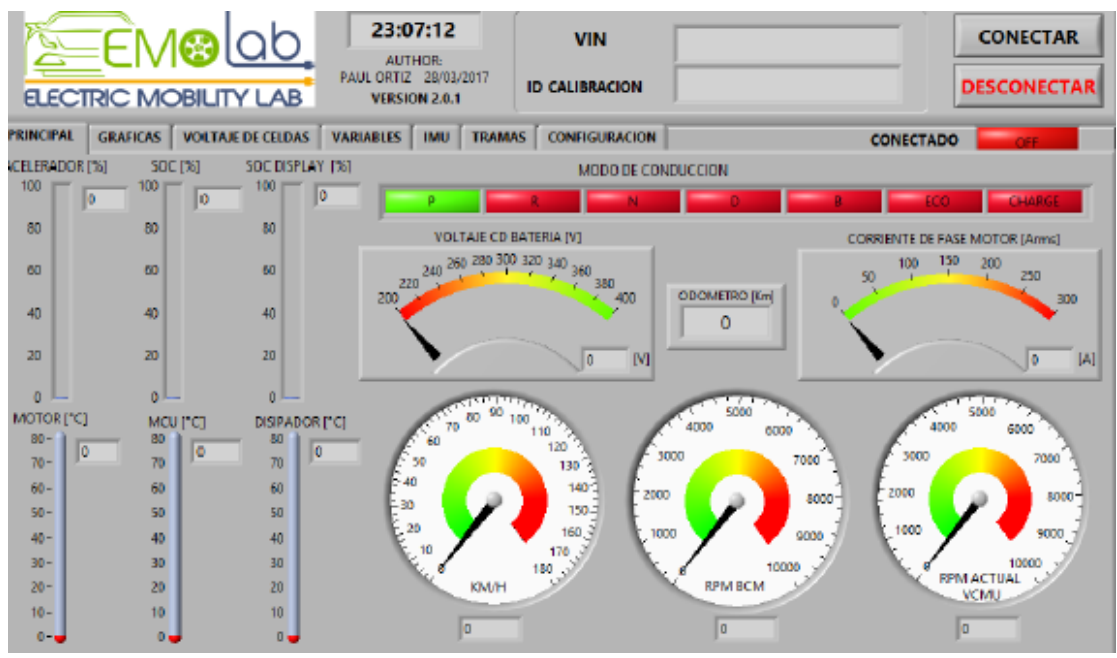

La medición de los parámetros de funcionamiento del vehículo eléctrico en cada uno de los ciclos típicos de conducción y con las respectivas cargas, se realizaron en el banco dinamométrico de chasis, en la figura 28 se observa los componentes del banco de potencia.

\section{Pruebas y recolección de datos}

La recolección de datos consiste en realizar cuatro pruebas por cada combinación de parámetros (peso y pendiente) en cada uno de los tres ciclos de conducción, dando como resultado la realización de 108 pruebas en total. La utilización del banco dinamométrico y el software EMOLAB V2.0.1 servirá para obtener los datos de consumo energético, simulando los diferentes ciclos de conducción ya mencionados anteriormente, además el banco de rodillos proporciona la función de simular la fuerza tractora a la que está sometido el vehículo en circulación.

Para la simulación de la fuerza tractora del vehículo en el banco de rodillos, se considera los parámetros (peso y pendiente) que afectan al consumo energético y la autonomía del mismo. Por lo tanto, para el desarrollo de las pruebas conociendo el peso del vehículo en vacío (1500 $\mathrm{kg}$ ), se estimó un peso por cada ocupante alrededor de los 70 a $75 \mathrm{~kg}$; adicionalmente se calculó la pendiente mínima, promedio y máxima de cada instante de tiempo que esta de manera implícita en cada uno de los ciclos de conducción.

Para el desarrollo de las pruebas se realiza un diseño experimental factorial (DOE) de dos niveles para determinar la influencia del peso y la pendiente como variables de entrada sobre el consumo energético y la autonomía del vehículo eléctrico como variables de salida y finalmente los datos obtenidos se analizan en el software Minitab. 


\section{Análisis de resultados y discusión}

\section{Consumo energético y autonomía real del vehículo eléctrico}

Para la determinación del consumo energético, se tomaron todas las muestras de forma aleatoria de cada uno de los tres ciclos de conducción y aplicando el diseño de experimentos factorial (DOE) se obtiene los efectos principales que se muestran en la figura 4 , donde se observa que el parámetro que mayor consumo energético genera es el tipo de ciclo de conducción, seguido por la pendiente a superar y en menor medida el peso de los ocupantes en el vehículo, en otras palabras, variables como el estilo de conducción, tráfico vehicular, estado de las vías, distancia y tiempo de recorrido (implícitos en el ciclo de conducción) tienen mayor influencia sobre el consumo energético que la pendiente a superar y el peso del vehículo.

\section{Figura 4}

Efectos principales para el consumo energético del vehículo eléctrico.

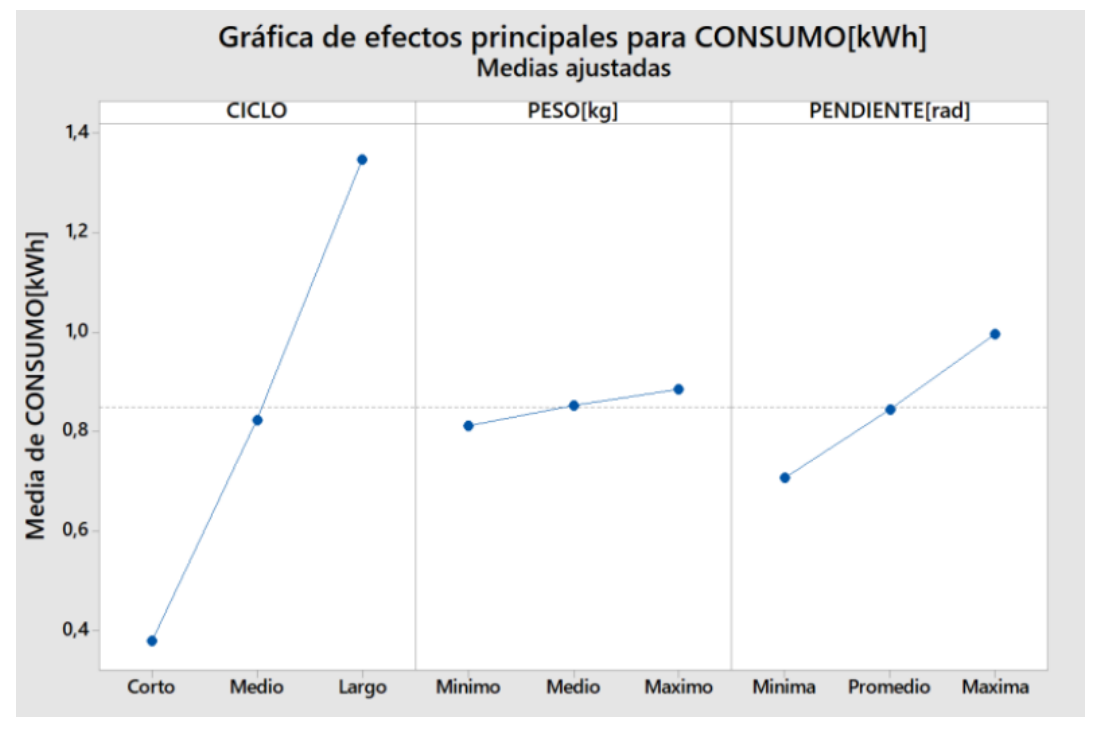

En la figura 5 se observa el efecto de la variación de cada uno de estos factores sobre la variable respuesta, teniendo así que a mayor distancia de recorrido y pendiente a superar el consumo de energía aumenta considerablemente, mientras que la velocidad promedio y el peso no inciden de manera significativa sobre el consumo energético. 


\section{Figura 5}

Efectos principales de las variables independientes sobre el consumo energético del EV.

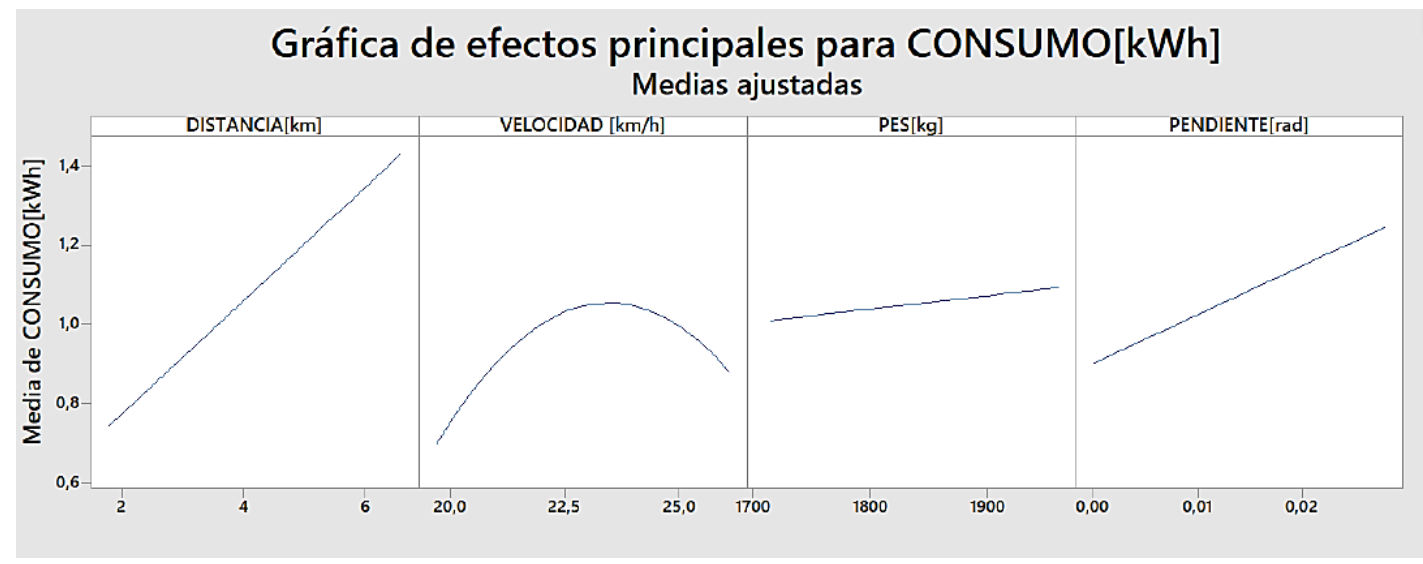

A mayor distancia de recorrido y pendiente a superar, el consumo energético incrementa también en magnitud de la forma que se observa en la figura 6.

\section{Figura 6}

Variación del consumo energético a diferentes distancias de recorrido y pendientes a superar.

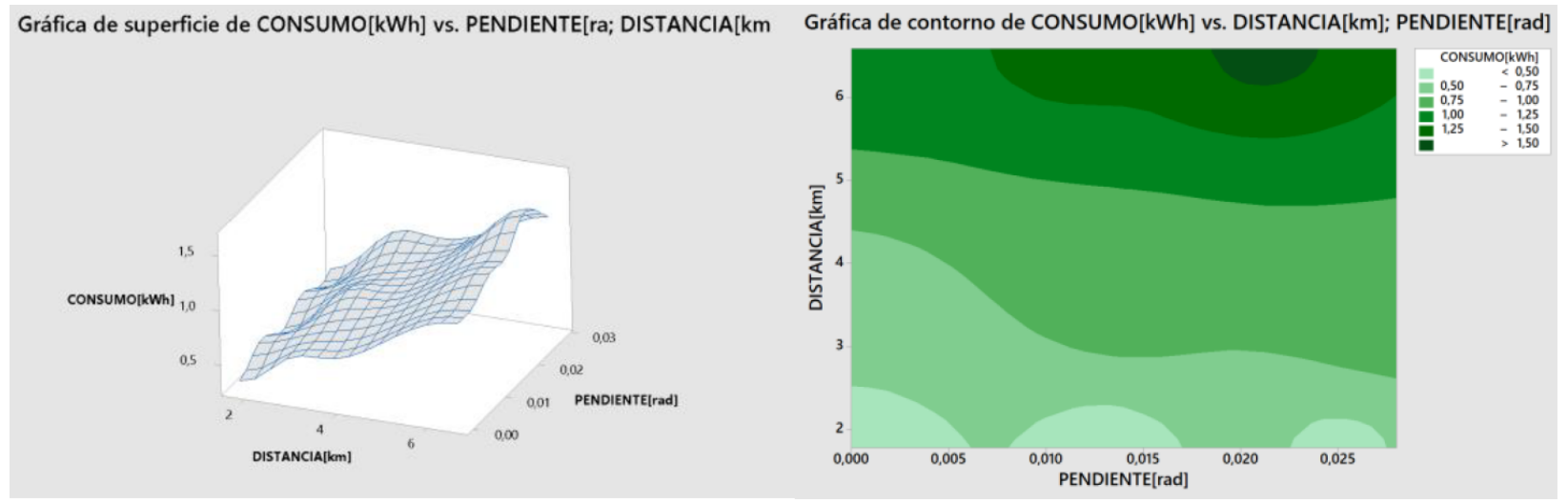

En la figura 7 se observa que la batería de $27 \mathrm{kWh}$ con la que está equipado el vehículo eléctrico actualmente, permite una autonomía con una distancia de recorrido menor a los $135 \mathrm{~km}$, mientras tanto que, para satisfacer una distancia de recorrido diario promedio de $200 \mathrm{~km}$, el vehículo debería poseer una batería con una capacidad mayor o igual a los $43 \mathrm{kWh}$. 


\section{Figura 7}

Capacidad energética requerida por la batería del vehículo eléctrico.

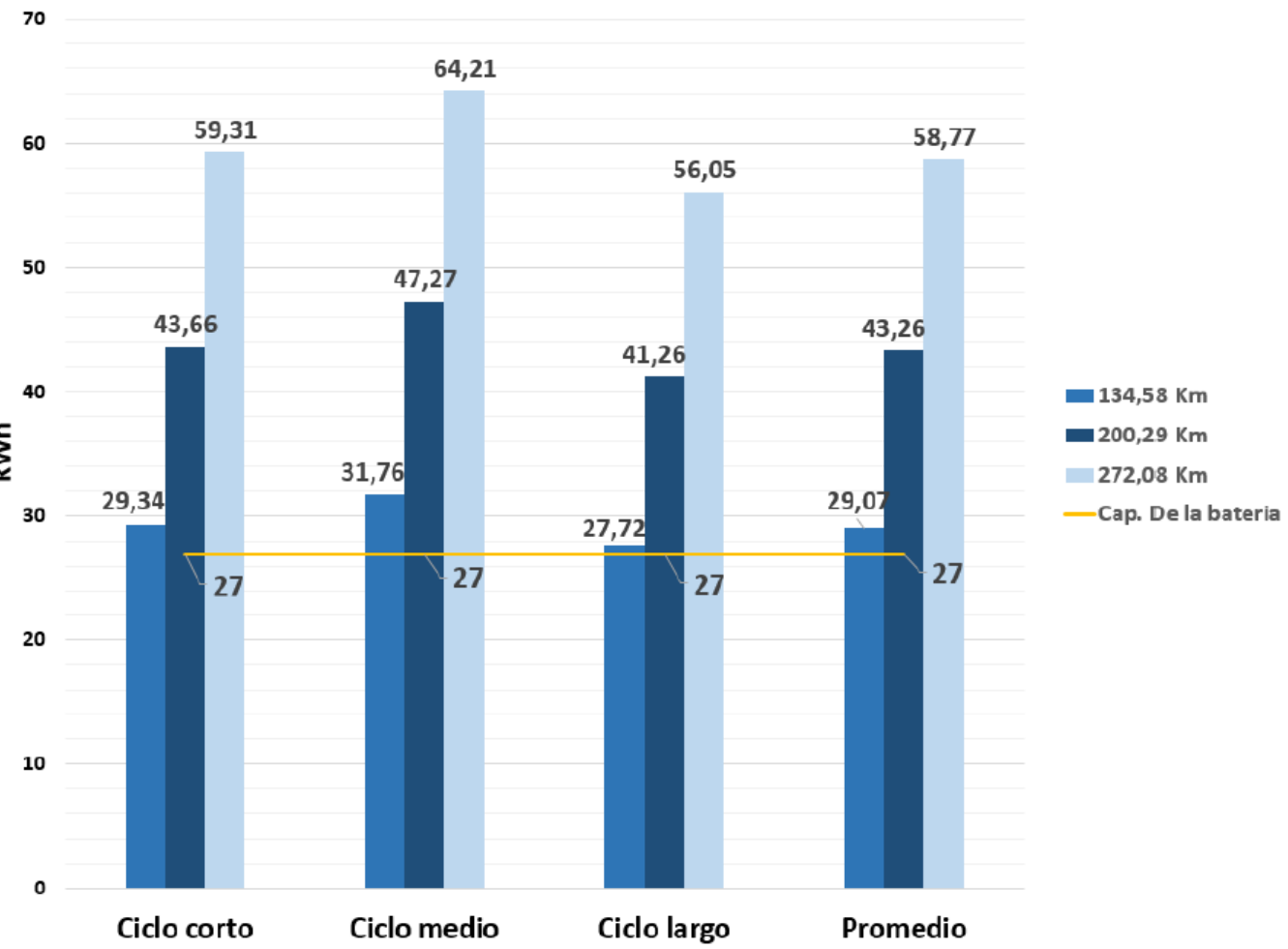

\section{Viabilidad económica del vehículo eléctrico}

Los resultados obtenidos se resumen en la tabla 3 y los casos son: Caso 1: Mantenimiento preventivo en taller oficial. Caso 2: Mantenimiento preventivo en taller independiente. Caso 3: Mantenimiento preventivo en taller independiente y sin gasto por sueldo del conductor.

\section{Tabla 3}

Resumen de la evaluación económica considerando los tres casos

\begin{tabular}{lcccccc}
\hline Vehículo & & VAN(USD) & TIR(\%) & $\boldsymbol{i ( \% )}$ & $\mathbf{R}_{\mathbf{b} / \mathbf{c}}$ & Viabilidad \\
\hline Soul EV & Caso 1 & -31950.27 & -0.14 & 10.21 & 0.71 & No \\
& Caso 3 & 18275.19 & 19 & 10.21 & 1.3 & $\mathrm{Si}$ \\
\cline { 2 - 7 } Accent & Caso 1 & -962.7 & 9 & 10.21 & 0.99 & $\mathrm{No}$ \\
& Caso 2 & 2542.80 & 12 & 10.21 & 1.02 & $\mathrm{Si}$ \\
\multirow{3}{*}{$\begin{array}{l}\text { Eveo } \\
\text { Emotion }\end{array}$} & Caso 3 & 52768.26 & 47 & 10.21 & 1.9 & $\mathrm{Si}$ \\
& Caso 1 & -5396.99 & 6 & 10.21 & 0.95 & $\mathrm{No}$ \\
& Caso 2 & -762.99 & 10 & 10.21 & 0.99 & $\mathrm{No}$ \\
& Caso 3 & 49462.47 & 44 & 10.21 & 1.8 & $\mathrm{Si}$ \\
\hline
\end{tabular}


Los resultados de la tabla 3 muestran que la inversión en un vehículo eléctrico es viable económicamente para el caso 3 ya que , y, siempre y cuando la autonomía $(125 \mathrm{~km})$ sea suficiente para cubrir el cupo de carreras mínimo (17 carreras, ver tabla 40) que permita obtener los ingresos para generar la rentabilidad que se observa en la tabla; lo cual no necesariamente es así, en otras palabras, la incertidumbre de si la autonomía alcanzará para cubrir ese cupo de carreras hace que el proyecto de inversión para el vehículo eléctrico (caso 3) sea de alto riesgo y como hay proyectos de inversión (vehículos Accent y Aveo) mucho más rentables y con riesgo relativamente más bajo, hacen que invertir en un Kia Soul EV para taxi sea descartable.

Para los tres vehículos analizados, la tabla 3 muestra que solo el caso 3 genera rentabilidad relativamente alta, lo cual significa que no resulta beneficioso invertir en un taxi si se va a contratar un conductor, al menos de forma permanente, excepto por el caso 2 para el Hyundai Accent, el cual sin embargo genera baja rentabilidad.

A pesar de los elevados costos en el vehículo eléctrico (inversión inicial y costo de la batería de alto voltaje), se puede inferir que el mayor problema para su implementación más que lo económico, pasa por el tema de la autonomía $(125 \mathrm{~km})$, ya que en dichas figuras se observa que se genera rentabilidad y recuperación de la inversión de forma relativamente rápida.

\section{Conclusiones}

Los consumos obtenidos con las pruebas realizadas en el banco dinamométrico mediante los diferentes ciclos de conducción son valores aproximados, ya que en el banco dinamométrico no se puede emular de forma exacta los comportamientos de manejo que se da en la ciudad, donde la circulación se ve afectada por frenadas y aceleraciones bruscas producidas por las acciones de los demás conductores o por el estilo de conducción del conductor del vehículo en cuestión.

Los valores de la autonomía y capacidad de la batería fueron obtenidos a raíz de los datos adquiridos mediante las pruebas realizadas con el software de ingeniería EMOLAB V2.0.1 en el banco dinamométrico de chasis con los diferentes ciclos típicos de conducción representativos de los taxis de Cuenca, lo cual demuestra una autonomía y capacidad de la batería inferior a las indicadas por el fabricante del vehículo.

Los resultados muestran una autonomía real para el vehículo eléctrico de $124.86 \mathrm{Km}$, con un consumo de energía promedio de $0.216 \mathrm{kWh} / \mathrm{km}$, equipado con una batería de $27 \mathrm{kWh}$, magnitudes que se contrarrestan con la necesidad de los taxis de Cuenca para satisfacer una distancia de recorrido diario promedio (KVR) igual a $200.29 \mathrm{~km}$, para lo cual el vehículo debería poseer una batería con una capacidad energética mayor o igual a los $43 \mathrm{kWh}$.

De acuerdo con los cálculos realizados se ha llegado a determinar los inconvenientes que presenta el vehículo eléctrico en cuanto a la batería de alta tensión, misma que no tiene la capacidad para almacenar la energía necesaria para el recorrido promedio, por lo que para cumplir con este recorrido necesariamente se tendría que hacer una recarga intermedia que perjudica la jornada laboral de los taxistas si el tipo de recarga no es rápido. 
Aunque el vehículo eléctrico en la actualidad cuenta con grandes ventajas sobre los vehículos con motor de combustión, como bajo costo por mantenimiento preventivo y costo de energía más barato, el desarrollo de este proyecto muestra que sigue teniendo las mismas desventajas [66] que ya tenía en el siglo XX, las cuales de momento no han sido superadas y son: elevado costo de producción y sobre todo baja autonomía.

Para solventar el problema de la autonomía sin incrementar la capacidad de la batería, se podría instalar masivos puntos de recarga rápida, siempre y cuando la degradación o reducción de la vida útil de las baterías no sea significativa por este tipo de recarga; sin embargo, de darse estas condiciones, la dificultad aquí seria: quien debe asumir este gasto de implementación de los puntos de recarga, siendo más un problema socioeconómico que técnico.

El propietario de taxi que vaya a renovar su unidad, debería invertir en un vehículo con motor de combustión, porque en las condiciones actuales la inversión en un vehículo eléctrico es de alto riesgo y cuenta con una limitada autonomía que no cumple con los requerimientos del sector, por lo tanto, no es recomendable un vehículo eléctrico tipo taxi en la modalidad convencional para Cuenca porque su viabilidad queda condicionada al requerimiento de cumplir con un mínimo de 17 carreras diarias con $125 \mathrm{~km}$ de autonomía.

\section{Referencias bibliográficas}

AEDIVE. (01 de 09 de 2017). Asociación empresarial para el desarrollo e impulso del vehículo eléctrico. Obtenido de http://aedive.es/taxis-electricos/

AMB. (01 de 09 de 2017). Àrea Metropolitana de Barcelona. Obtenido de http://taxi.amb.cat/s/es/imet/taxi-sostenible/punts-de-recarrega-d-energia-alternativa.html

Ambiente, S. D. (01 de 09 de 2017). Alcaldía Mayor de Bogotá. Obtenido de http://ambientebogota.gov.co/web/taxis-electricos-en-bogota/que-son-los-taxis-electricos

ANT. (2009). Resolución No. 006-B-DIR-2009-CNTTTSV. Quito: Agencia Nacional de Tránsito. ANT. (2015). Resolución No. 053-DIR-2015-ANT. Quito: Agencia Nacional de Tránsito.

Cárdenas, J. (01 de 09 de 2017). EL ESPECTADOR. Obtenido de http://www.elespectador.com/noticias/bogota/taxis-electricos-se-quedan-sin-energia-ysin-respaldo-articulo-682709

Chuquiguanga, W., Jiménez, B., \& Méndez, P. (2018). Análisis de viabilidad para la implementación de un vehículo eléctrico que preste el servicio de taxi en la ciudad de cuenca. Cuenca: UPS.

COMUNICADOS.CO. (01 de 09 de 2017). COMUNICADOS.CO. Obtenido de https://comunicados.co/2017/07/taxis-electricos-podrian-tener-una-segunda-oportunidadde-rodar-en-bogota/

González, E. O. (2015). Viabilitat de la implementació d'una xarxa de taxis elèctrics a Barcelona. Barcelona: UPC BARCELON.

Gonzalez, J. P. (2017). EMOLAB (Programa de ordenador software). Madrid: Patente CUE003106.

Guarnieri, M. (2012). Looking Back to Electric Cars. Padova: IEEE Xplore Digital Library. Izquierdo, A. (1995). Teoría de los vehículos automóviles. Madrid. 
J. D. T. Sarmiento. (2015). Estudio de Viabilidad en la implementación de vehículos eléctricos en la ciudad de Cuenca. Cuenca: Universidad Politécnica Salesiana.

J. L. D. Fernández. (2013). Análisis de la viabilidad del vehículo eléctrico en una empresa de reparto de comida a domicilio. Barcelona: Universitat Politècnica de Catalunya.

Loaiza, S. C. (2016). Obtención de los parámetros necesarios para el cálculo de la fuerza en rueda de vehículos eléctricos. Cuenca: Universidad del Azuay.

Maxi, J. C. (2014). Diseño de un modelo de Costos alternativo para la determinación de la tarifa de taxi modalidad convencional en la ciudad de Cuenca para el año 2014. Cuenca: universidad del Azuay.

MCPEC. (03 de 09 de 2017). Ministerio Coordinador de Producción, Empleo y Competitividad. Obtenido de Gobierno Nacional de la República del Ecuador: http://www.produccion.gob.ec/vehiculos-electricos-una-realidad-en-ecuador/

Moncayo, D. S. (2016). Determinación del indicador kilómetros-vehículo recorrido (KVR) para la ciudad de Cuenca. Cuenca: Universidad Politécnica Salesiana.

Mora, J. F. (2008). Convertidores electrónicos de potencia. Madrid: McGraw-Hill.

Mourik, L. V. (03 de 09 de 2017). FuturENERGY. Obtenido de http://www.futurenergyweb.es/pdf/articulos/2015-12/FuturEnergy-Dic15_71-75.pdf

movilidadeléctrica.com. (03 de 09 de 2017). movilidadeléctrica.com. Obtenido de https://movilidadelectrica.com/amsterdam-ciudad-sostenible/

MUNICPAL, E. C. (2016). Ordenanza que crea y regula el servicio de taxi. Loja: Municipio de Loja.

R. G. Déniz, R. J. (2013). Estudio para la implantación del Vehículo Eléctrico en Canarias. Las Palmas: Instituto Tecnológico de Canarias.

Sibri, V. G. (2012). Estudio de la vida útil de los vehículos desde la perspectiva de la gestión de mantenimiento del transporte público modalidad taxis de la ciudad de Cuenca. Cuenca: Universidad Politécnica Salesiana. 